\title{
Real-time vascular mechanosensation through ex vivo artery perfusion
}

\author{
Rahul M Prasad ${ }^{1,2}$, Xingjian Jin ${ }^{1}$, Wissam A AbouAlaiwi ${ }^{1}$ and Surya M Nauli ${ }^{1,2,3^{*}}$
}

\begin{abstract}
Background: Cell-based perfusion studies have provided great insight into fluid-sensing mechanisms, such as primary cilia in the renal and vascular systems. However, the intrinsic limitations of in vitro cell culture, such as the inability to reflect cellular organization within tissues, has distanced observed paradigms from possible clinical developments. Here we describe a protocol that applies ex vivo artery perfusion and calcium imaging to observe real-time cellular responses to fluid-shear stress.

Results: Through our ex vivo artery perfusion method, we were able to simulate physiological flow and initiate distinct fluid shear stress mechanosensory responses, as well as induced acetylcholine responses in mouse aortic tissue. The observed calcium profiles confirm results found through previous in vitro cell culture experiments. The overall procedure, including dissection, sample preparation and perfusion, takes around 3 hours to complete.

Conclusion: Through our unique method, we are able to induce laminar flow within intact mouse aortic tissue and illicit subsequent cellular responses. This method of ex vivo artery perfusion provides the opportunity to bridge the novel findings of in vitro studies with subsequent physiological models of fluid-shear stress mechanosensation in vascular tissues.
\end{abstract}

\section{Background}

Mechanosensation of blood flow is a crucial function of the vascular system. Defects in the ability to adequately sense and respond to blood flow have been linked to several cardiovascular pathologies including hypertension, atherosclerosis, and aneurysms [1]. Through the use of in vitro assays, which utilize fluid perfusion of cultured cells, numerous insights have been made into the mechanisms of flow sensation such as the role of the primary cilium, endothelial glycocalyx, cytoskeleton and many others.

Despite breakthroughs in identifying mechanisms of blood flow, the in vitro perfusion system remains limited in its application to understanding and treating disease states by their inability to replicate crucial in vivo influences. The diversity of cellular populations within blood vessels is an excellent example of this; current cell culture technologies are not capable of emulating the

\footnotetext{
*Correspondence: Surya.Nauli@UToledo.Edu

${ }^{1}$ College of Pharmacy and Pharmaceutical Sciences, The University of Toledo, Toledo, $\mathrm{OH}$, USA

${ }^{2}$ College of Medicine and Life Science, The University of Toledo, Toledo, $\mathrm{OH}$, USA

Full list of author information is available at the end of the article
}

organization of several cell types. The organization of cell-types have been shown to be essential to such functions as remodeling in atherosclerosis models as well as cell-cell communication, as is the case between endothelial cells and vascular smooth muscle cells through nitric oxide $[2,3]$. In addition to this, the physical structure of vascular tissue remains elusive to in vitro approaches. Local hemodynamic forces in vascular tissue, produced by curvatures, lumen diameter, and branch points, have been shown to have profound effects in the form of gene expression and pathogenesis [4]. While in vitro studies have been able to simulate and confirm the effects of some of these forces, such as shear stress, ex vivo studies remain the most physiologically relevant due to their ability to observe the combined effects of nearly all the fluid forces acting on the cells.

Due to the breath of literature citing intracellular $\mathrm{Ca}^{2+}$ as a secondary messenger, the use of fluorescent calcium indicators is chosen as the means of observing endothelial sensory function. Within endothelial cells, intracellular $\mathrm{Ca}^{2+}$ has been shown to be vitally involved in the maintenance of tone, nitric oxide synthesis, ion channel regulation, and triggering of growth mechanisms. Intracellular $\mathrm{Ca}^{2+}$ has 
also been established as an read-out of monitoring fluid flow sensation in numerous other cell types including stem cells [5], renal epithelial [6], hepatic epithelial [7], pancreatic duct [8], nodal epithelial [9], dentin [10], osteoblasts and osteocytes [11,12].

In our study, we developed a method for observing fluid-shear stress mechanosensation in mouse aortic explants (Figure 1). Although ex vivo perfusion studies have been conducted on vascular tissues, our method provides the novel ability to deliver and induce mechanosensory vascular responses unique to fluid shear stress [13-18]. The experimental method we describe provides the ability to induce laminar flow through a cannulated artery without damaging the endothelia, maintain the artery in a physiologically relevant environment, and clearly visualize the tissue (Figures 2 and 3 ).

\section{Results and discussion}

The described methodology allows for the observation of cellular responses to fluid flow. The calcium response profile for induced fluid flow in wild type mouse tissue (Figure 4) is very similar to the extensively characterized profile of endothelial in vitro responses [19,20]. This response is characterized by a rapid increase in intracellular calcium, peaking after approximately 5 seconds of induction, and then plateau below the baseline. The similarity of the profiles suggests that the readout is indicative of the shear stress response transduced by endothelial cilia. In addition to the fluid flow response, the profile for tissue induced by acetylcholine again matches those of endothelial in vitro responses $[19,20]$. This response is distinguished in both systems as a rapid increase within 5 seconds, at a slightly higher level than that of fluid flow alone, and a sustained elevation of intracellular calcium. As the response to acetylcholine has been widely characterized, it is used as a control for viability.

Based on lumen diameter, we used a flow rate to induce a shear stress of $7.2 \times 10^{4} \mathrm{~kg} / \mathrm{m} / \mathrm{sec}^{2}$, as this has been shown in in vitro studies to be the optimal level of endothelial shear stress response $[19,20]$. We found that at least 3 flow experiments were able to be conducted before the tissue was rendered unviable. Importantly, our set-up confirms that our tissue was in focus throughout our experimental procedure (Additional files 1 and 2: Movies 1 and 2). In addition, the differential interference contrast movies also indicate a stably positioned tissue within our set-up, suggesting no abrupt change in pressure or disruption was observed during our experiment.

While the primary advantage of our method is the ability to observe vascular mechanosensory responses to shear stress, the system can be adapted for other appropriate applications. For example, pressure sensing is a common approach for recording responses through tension changes as a result of vasoconstriction or dilation in blood vessels [21]. In addition, the potential for ex vivo perfusion to study the etiology of atherosclerosis has recently arisen [18]. Similar to the use of fluorescence imaging to observe responses of the vascular endothelial or smooth muscle cells, other groups have used fluorescence imaging to observe leukocyte adhesion to the endothelium of perfused vessels - a crucial process in the pathogenesis of atherosclerosis $[15,17,18]$. Finally, ex vivo artery perfusion has also been utilized as a means of drug delivery development [16].

\section{Conclusion}

In order to apply the insights of in vitro studies to physiological models, we have developed ex vivo artery perfusion technology to allow for the measurement of the cellular response as a result of fluid-shear stress mechanosensation. This approach may serve as the next step in applying in vitro findings in a more physiologically relevant manner and in investigating the mechanisms of vascular mechanosensation - a crucial measure towards developing therapeutics for vascular disease.

\section{Materials \\ Reagents}

Adult mouse; distilled water; DMEM (Thermo Scientific, cat. no. SH30243.01); Fluo-2 AM (TEFLabs, cat. no. 0222);

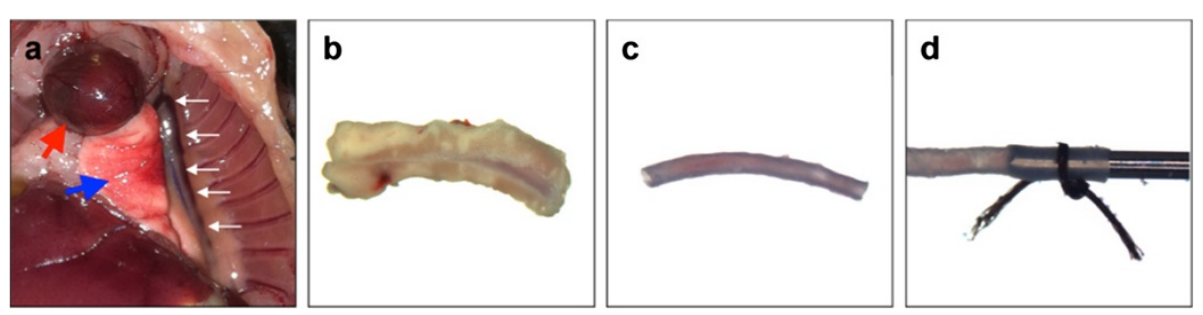

Figure 1 Dissection, preparation and cannulation of mouse vascular tissue. In this figure, mouse thoracic aorta is shown as an example. a. Mouse thoracic cavity is exposed, and heart (red arrow) is displaced to better reveal the lung (blue arrow) and aorta (white arrows). b. After the aorta is dissected out from the mouse, blood is perfused out from the lumen with a 30 gauge needle. The encapsulation of vessel by fat and connective tissue is visible. $\mathbf{c}$. Aorta is cleaned by removing the surrounding tissues. $\mathbf{d}$. The aorta is then cannulated and tied with silk suture to form a surgeon's knot. 

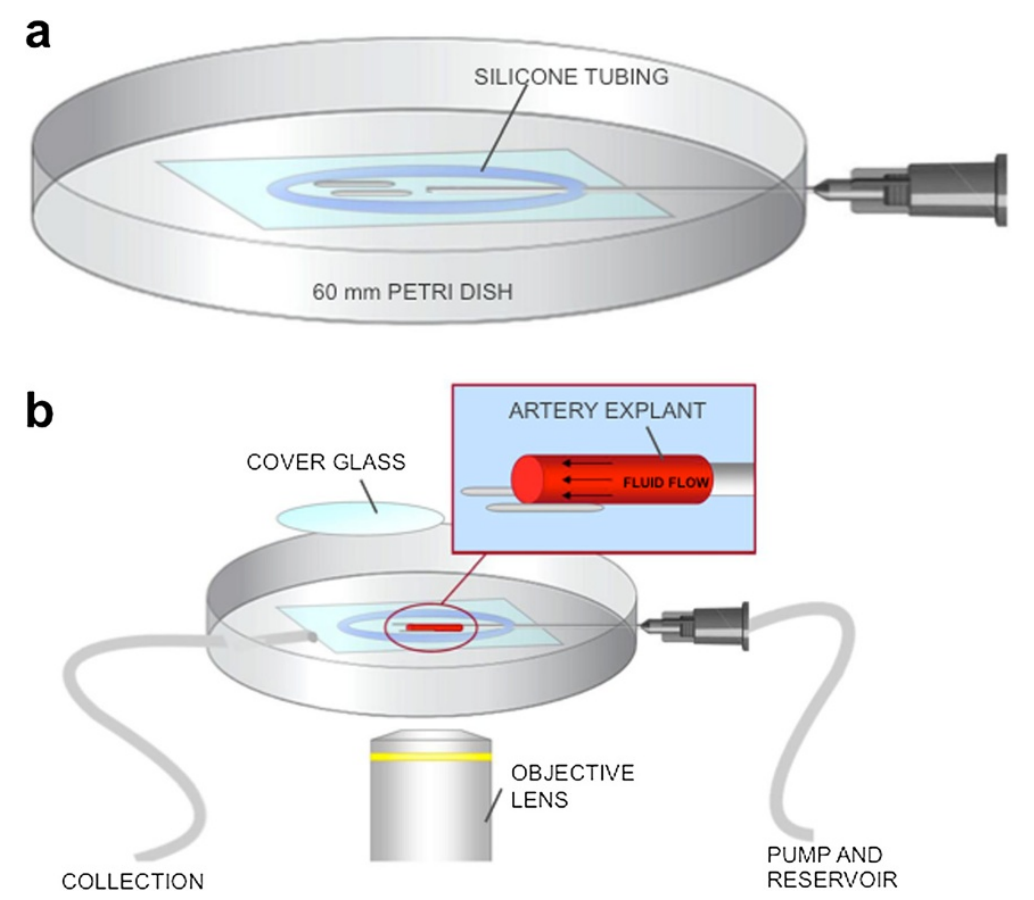

Figure 2 Schematic representation of perfusion apparatus. a. An inexpensive apparatus can be made using a 100-mm cell culture dish, a 22-g hypodermic needle, glass capillary tubes, a glass cover slip, silicone tubing, and non-cytotoxic epoxy and silicone glues. b. After cannulation to the apparatus, the vessel is covered with a cover glass. The chamber is placed on the microscope and connected with the rest of perfusion system.

acetylcholine chloride (Sigma, a2661-25 g); dimethyl sulfoxide (Sigma, d8779); ethanol, 95\%, vol/vol (PharmcoAAPER, 111000190); silicone (GE, stock no. GE50090442); epoxy (Henkel, co. no. 1393760).

\section{Equipment}

Sterile dissection instruments: forceps (Roboz Surgical), scissors (Roboz Surgical); culture dishes, $100 \mathrm{~mm}$ (Thermo Scientific cat. no. 130182); syringe, $1 \mathrm{ml}$ (BD Luer-Lok, cat. no. 309628); Syringe, $30 \mathrm{~mL}$ - Luer (Heinke-Sass-Wolf, cat. no. 4830001000); hypodermic needles, 22 gauge (BD PrecisionGlide, cat. no. 305156); hypodermic needles, 30 gauge (BD PrecisionGlide, cat. no. 305106); sutures 5-0 (Teleflex Medical); microscope slides (Fisherbrand, cat. no. 451000); coverslips, $22 \mathrm{~mm} \times$ $32 \mathrm{~mm}$ (VWR International, cat. no. 831-0134); capillary, outside diameter of $1.5 \mathrm{~mm}$, thickness of $0.2 \mathrm{~mm}$ (Kimble, Inc.); humidified, water-jacketed incubator (Binder) at $37^{\circ} \mathrm{C}$ and $5 \% \mathrm{CO}_{2}$; dissecting microscope (Zeiss, Stemi SV6); Nikon microscope (Eclipse, TE2000$\mathrm{U})$; Photometric monochrome camera (Coolsnap EZ $20 \mathrm{MHz}$ ); light box (Zeiss); perfusion pump; Nalgene 180 PVC tubing, 1/32 in. I.D., 3/32 in. O.D., 1/32 in. wall (Thermo Scientific, part no. 8000-002); $\mathrm{CO}_{2}$ gas tank with regulator.

\section{Methods}

\section{Reagent setup}

Ethanol (70\%, vol/vol) was prepared by diluting absolute ethanol in distilled water and store indefinitely at room temperature. $50 \mu \mathrm{g}$ of Fluo- $2 \mathrm{AM}$ was dissolved in $50 \mu \mathrm{L}$ of dimethyl sulfoxide, distributed into $5 \mu \mathrm{L}$ aliquots and stored indefinitely at $-20^{\circ} \mathrm{C}$. A variety of buffers may be used to may be used to bathe and perfuse the tissue samples - we chose HEPES-based DMEM (see Reagents). Buffers were stored at $4^{\circ} \mathrm{C}$ until use. Before perfusion, the buffer was warmed at $37^{\circ} \mathrm{C}$ for $30 \mathrm{~min}$. Throughout our experiment, we used DMEM because it has similar mechanic properties as blood plasma. In addition, DMEM acts as Newtonian fluid making it easier in our fluid-shear stress calculation. For pharmacological reagents, a stock solution was made in distilled water and at an appropriate volume to a volume of buffer for the desired concentration. The final solution was loaded into the perfusion syringe prior to treatment. For acetylcholine, we made a $100 \mathrm{mM}$ stock solution, then added $40 \mu \mathrm{L}$ of the stock to $40 \mathrm{~mL}$ of DMEM for a final concentration of $10 \mu \mathrm{M}$.

\section{Equipment setup}

Mice were housed in pathogen-free, regulated-humidity conditions and kept in a 12-h light cycle with sterilized 


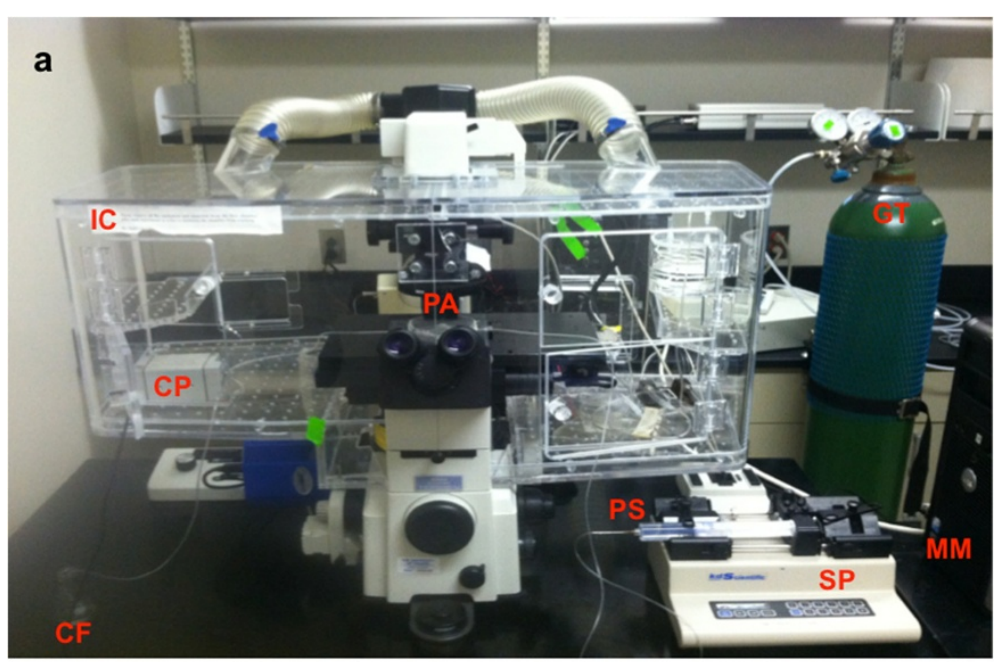

b

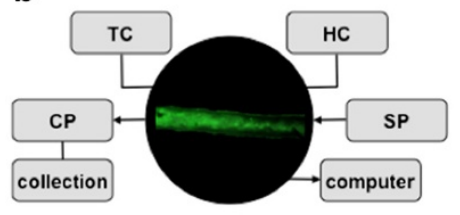

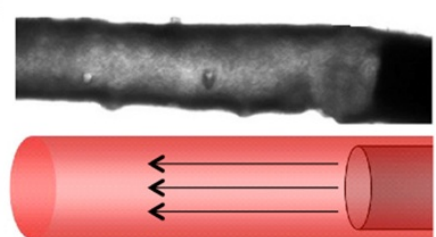

Figure 3 Overview of experimental system. An ex vivo perfusion fluid-flow setup is shown to maintain a healthy functional tissue for the duration of experiment. a. The setup includes a computer with MetaMorph software (MM), syringe pump (SP), perfusion syringe (PS), perfusion apparatus (PA), collection pump (CP), collection flask (CF), incubation chamber (IC), gas tank (GT). b. Not visible in picture is a temperature controller ( $\mathrm{TC}$ ), humidity controller ( $\mathrm{HC}$ ), and computer monitor. Artery segment has a baseline fluorescent after loaded with calcium indicator. c. Brightfield image of the artery segment is visualized. As depicted by the diagram below, a perfusion needle is cannulated to the artery to deliver fluid-flow.

chow and water provided. The 'Three Rs' (replacement, reduction, refinement) were observed in the design and execution of all experiments. The use of animal cells or tissues was approved by the Animal Care and Use Committee of The University of Toledo. We have complied with all the governmental and institutional guidelines for the care and use of animals within our research program. All dissection instruments were kept in $70 \%$ ( $\mathrm{vol} / \mathrm{vol}$ ) ethanol during the procedure to maintain sterility.

A Nikon TE2000-U was connected with a photometric monochrome camera (Coolsnap EZ $20 \mathrm{MHz}$ ). A high speed excitation wavelength changer for DG4/DG5
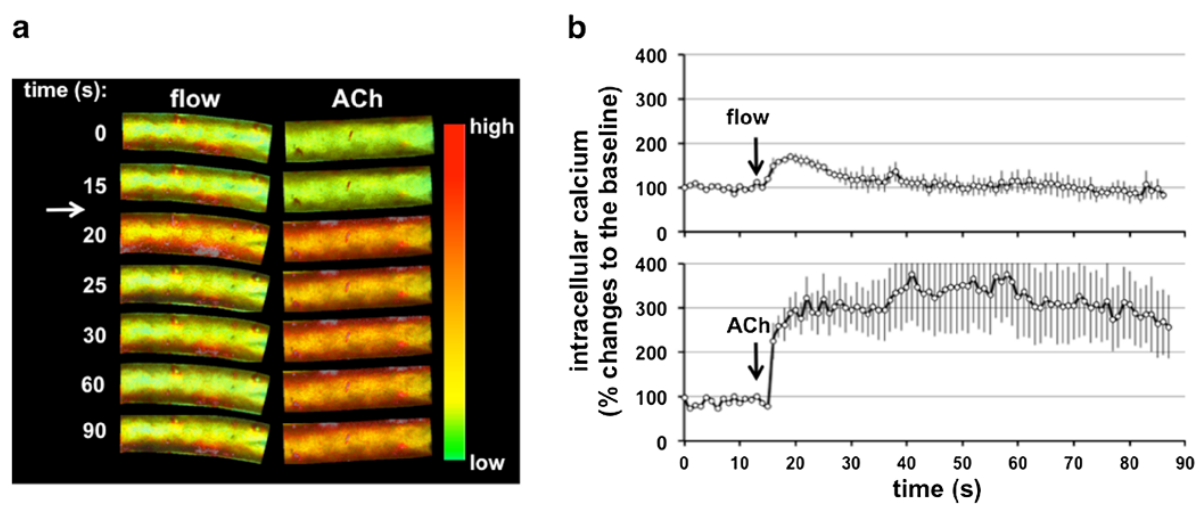

Figure 4 Distinct calcium responses to fluid-shear stress and a pharmacological agonist. After loaded with calcium indicator, a segment of artery is imaged under an inverted microscope. Baseline calcium level is recorded for at least 15 seconds (s). a. Pseudocolor indicates intracellular calcium level in response to fluid-shear stress in the presence or absence of acetylcholine (ACh). Green to red color indicates low to high calcium level, respectively. $\mathbf{b}$. Changes in calcium level in response to flow or acetylcholine are quantified and normalized relative to baseline calcium level. Arrows indicate the initiation of perfusion. 
system was used to capture at DIC and a fluorescence excitation wavelength of $380 \mathrm{~nm}$, under the control of MetaFluor / MetaMorph software.

\section{Building the perfusion apparatus}

A glass bottom dish was used to make the perfusion apparatus, as the plastic polymers used in other culture dishes interferes with the recording of fluorescence (Figure 2). To make this, a circular hole was made in the center of a $100 \mathrm{~mm}$ cell culture dish and Epoxy was used to glue a coverslip to cover the hole (see Materials). Using small diameter silicone tubing (Thermo Scientific Nalgene 180 PVC tubing) a $35 \mathrm{~mm}$ closed ring was then formed. The ring was around the glass bottom center of the dish, using silicone. The silicone was left to dry for 2 hours, forming a sealed barrier to prevent leakage. A slit in the culture dish was made, which was wide enough to rest the bevel of the needle on. The tip of a $22 \mathrm{~g}$ needle was removed and blunted. The needles was then passed through the slit toward the middle of the ring, piercing through the silicone in the process. The needle was angled at no more than 3 degrees from the bottom of the dish. Using Epoxy, the needle was glued in place, allowing the epoxy to dry for 2 hours. In order to prevent the tissue from swaying during perfusion, two pieces of capillary tube were taped across from the tip of the perfusion needle.

\section{Collection and preparation of aortic tissue}

First, a mouse was euthanized through $\mathrm{CO}_{2}$ asphyxiation. As always, all animal experiments complied with national laws and institutional regulations. In a tray with paper towels, the animal was positioned on its back to dissect the aorta. A transverse incision was made to open the abdominal cavity, followed by cutting of the diaphragm. Incisions where then made up the chest wall to the first or the second rib on both sides. A final incision was made along the sternum to expose the heart. The aorta was exposed by displacing the lungs and intestines, and is adhered to the vertebral column through fatty connective tissue. After locating the aorta, the arch of the aorta is decannulated from the heart, brachiocephalic, carotid and subclavian arteries. In a cranial to caudal direction, the thoracic and abdominal aorta was slowly separated from the vertebral column. Finally, the abdominal aorta was decannulated from the distal aorta.

In order to remove intraluminal blood, the tissue was placed in ice cold oxygenated DMEM and slowly perfused with $0.5 \mathrm{~mL}$ of DMEM. We found that perfusion must be conducted gently and no more than twice - multiple or high pressure perfusion will damage the endothelium of the vessel and render the tissue unresponsive. After removing the intraluminal blood, fatty connective tissue surrounding the vessel was gently cut away, taking care to prevent large nicks and holes in the tissue.

A 5 microliter aliquot of Fluo-2 calcium dye was diluted with 250 microliters of DMEM in a centrifuge tube. The tissue sample was then placed in centrifuge tube. The centrifuge tube was then incubated for 30 minutes in a $\mathrm{CO}_{2}$ incubator. Following incubation, the tissue was carefully removed placed in a centrifuge tube containing 500 microliters of DMEM, and then incubated for 10 minutes.

\section{Preparing and connecting the perfusion pump}

The organ bath chamber of the apparatus was filled with $4 \mathrm{~mL}$ of oxygenated warm DMEM, and the aorta sample was placed within in the organ bath. Prior to cannulation of the sample with the perfusion needle, $1 \mathrm{~mL}$ of perfusion solution was gently pipeted onto and through the connection port of the perfusion apparatus, thereby preventing air bubbles.

Using microforceps, the aorta was cannulated by sliding the sample over the needle. A small nick was made in the tissue, past the tip of the perfusion needle, in order to relieve the negative pressure inside the tissue that is generated automatically following cannulation. Excessive stretching will trigger stretch activated calcium channels and will ruin the integrity of the sample. After cannulation, a glass coverslip was taped over the organ bath chamber. We found that if a coverslip is not taped, the increase in fluid level as a result of perfusion causes a drastic drop in recorded fluorescence. The apparatus was then placed on the microscope stage. The tissue was left to equilibrate undisturbed in the apparatus for 15 minutes and but no longer than 30 .

In order to prepare the perfusion pump, the perfusion tubing was first placed into the perfusion pump. Warmed, oxygenated DMEM was pre-perfused through the clean silicone tubing. The perfusion pump tubing was then connected to the perfusion apparatus and the speed of perfusion was set on the pump.

\section{Perfusion and real-time fluorescence imaging}

Metamorph imaging software was used to record fluorescence levels of the sample. Channels of DIC and GFP (with excitation and emission filters of $400 \sim 430 \mathrm{~nm}$ and $508 \sim 520 \mathrm{~nm}$, respectively) were simultaneously captured with $1.07 \mathrm{~s}$ per frame. After recording a baseline of at least 15 seconds, the perfusion pump, with speed pre-set, was turned on. After 91 frames, perfusion was stopped. To prevent excess build up of media spilling out of the silicon ring within the perfusion apparatus, an additional pump and tubing can be attached to the apparatus in parallel and outside the ring. 


\section{Data analysis}

The captured images were compiled into a sequential stacked file. The background of the perfusion experiment was logged by selecting an identical region of the image plane, where no part of the sample overlapped. The average intensity of the region was logged to Microsoft Excel. Similarly, to analyze the sample, a region of the image plane was selected with only the sample contained. In cases where significant movement of the sample was observed, the region of data collection was appropriately adjusted on a frame by frame basis. The average intensity of the sample region was logged to the same Microsoft Excel file as the background values. The recorded fluorescence values of the background region were subtracted from the values of the tissue sample region. This allowed for fluorescence changes due to noise to be accounted for.

An average was calculated from the first fifteen data points of the new, adjusted values. This value was used as the recorded baseline, as this was the average intensity of the tissue prior to perfusion. The entire data set was then normalized by dividing all of the adjusted data set values by the calculated averaged baseline. The normalized data points were plotted on a graph to produce a representative trace. At least three experiments under each condition - perfusion and treatment with ACh. The data sets were respectively averaged and plotted with error bars indicated standard error.

\section{Additional files}

Additional file 1: Movie 1. Flow induced a transient intracellular calcium response in mouse artery ex vivo. Differential interference contrast (left) and fluorescence (right) movies were captured simultaneously at 1 frame per second. Number indicates time in second. Color bar indicates pseudocolor range where purple and white represent lowest and highest intracellular calcium levels, respectively.

Additional file 2: Movie 2. Acetylcholine induced a sustained increase in intracellular calcium in mouse artery ex vivo. Differential interference contrast (left) and fluorescence (right) movies were captured simultaneously at 1 frame per second. Number indicates time in second. Color bar indicates pseudocolor range where purple and white represent lowest and highest intracellular calcium levels, respectively.

\section{Competing interests}

The author's claim no competing financial interests.

\section{Authors' contributions}

Developing original approach (WAA, SMN), improving and confirming the technique (RMP, XJ), performing experiment (RMP), analyzing data (RMP, XJ), writing the manuscript (RMP, SMN). All authors read and approved the final manuscript.

\section{Acknowledgements}

This work was supported by the National Institute of Health DK080640 and the Department of Defense PR130153. Authors wish to thank Shirley Mei for her assistance in preparing the illustrations.

\section{Author details}

'College of Pharmacy and Pharmaceutical Sciences, The University of Toledo, Toledo, OH, USA. ${ }^{2}$ College of Medicine and Life Science, The University of Toledo, Toledo, OH, USA. ${ }^{3}$ Department of Pharmacology; MS 1015, Health Education Building; Room 274, The University of Toledo, 3000 Arlington Ave, Toledo, $\mathrm{OH} 43614$, USA.

Received: 9 January 2014 Accepted: 22 March 2014

Published: 31 March 2014

\section{References}

1. Ando J, Yamamoto K: Flow detection and calcium signalling in vascular endothelial cells. Cardiovasc Res 2013, 99:260-268.

2. Hahn C, Schwartz MA: Mechanotransduction in vascular physiology and atherogenesis. Nat Rev Mol Cell Biol 2009, 10:53-62.

3. Chatzizisis YS, Coskun AU, Jonas M, Edelman ER, Feldman CL, Stone PH: Role of endothelial shear stress in the natural history of coronary atherosclerosis and vascular remodeling: molecular, cellular, and vascular behavior. J Am Coll Cardiol 2007, 49:2379-2393.

4. Huo Y, Guo X, Kassab GS: The flow field along the entire length of mouse aorta and primary branches. Ann Biomed Eng 2008, 36:685-699.

5. Riddle RC, Taylor AF, Genetos DC, Donahue HJ: MAP kinase and calcium signaling mediate fluid flow-induced human mesenchymal stem cell proliferation. Am J Physiol Cell Physiol 2006, 290:C776-C784

6. Nauli SM, Rossetti S, Kolb RJ, Alenghat FJ, Consugar MB, Harris PC, Ingber DE, Loghman-Adham M, Zhou J: Loss of polycystin-1 in human cyst-lining epithelia leads to ciliary dysfunction. J Am Soc Nephrol 2006, 17:1015-1025.

7. Masyuk Al, Masyuk TV, Splinter PL, Huang BQ, Stroope AJ, LaRusso NF: Cholangiocyte cilia detect changes in luminal fluid flow and transmit them into intracellular Ca2+ and cAMP signaling. Gastroenterology 2006, 131:911-920.

8. Sankar KS, Green BJ, Crocker AR, Verity JE, Altamentova SM, Rocheleau JV: Culturing pancreatic islets in microfluidic flow enhances morphology of the associated endothelial cells. PLoS One 2011, 6:e24904.

9. McGrath J, Somlo S, Makova S, Tian X, Brueckner M: Two populations of node monocilia initiate left-right asymmetry in the mouse. Cell 2003, 114:61-73.

10. Lim KT, Kim J, Seonwoo H, Chang JU, Choi H, Hexiu J, Cho WJ, Choung PH, Chung JH: Enhanced osteogenesis of human alveolar bone-derived mesenchymal stem cells for tooth tissue engineering using fluid shear stress in a rocking culture method. Tissue Eng Part C Methods 2013, 19:128-145.

11. Xiao Z, Zhang S, Mahlios J, Zhou G, Magenheimer BS, Guo D, Dallas SL, Maser R, Calvet JP, Bonewald L, Quarles LD: Cilia-like structures and polycystin-1 in osteoblasts/osteocytes and associated abnormalities in skeletogenesis and Runx2 expression. J Biol Chem 2006, 281:30884-30895.

12. Delaine-Smith RM, Sittichokechaiwut A, Reilly GC: Primary cilia respond to fluid shear stress and mediate flow-induced calcium deposition in osteoblasts. FASEB J 2014, 28:430-439.

13. Meininger GA, Zawieja DC, Falcone JC, Hill MA, Davey JP: Calcium measurement in isolated arterioles during myogenic and agonist stimulation. Am J Physiol 1991, 261:H950-H959.

14. Dora KA, Doyle MP, Duling BR: Elevation of intracellular calcium in smooth muscle causes endothelial cell generation of NO in arterioles. Proc Natl Acad Sci U S A 1997, 94:6529-6534.

15. Woollard KJ, Suhartoyo A, Harris EE, Eisenhardt SU, Jackson SP, Peter K, Dart AM, Hickey MJ, Chin-Dusting JP: Pathophysiological levels of soluble Pselectin mediate adhesion of leukocytes to the endothelium through Mac-1 activation. Circ Res 2008, 103:1128-1138.

16. Hitchcock KE, Caudell DN, Sutton JT, Klegerman ME, Vela D, Pyne-Geithman GJ, Abruzzo T, Cyr PE, Geng YJ, McPherson DD, Holland CK: Ultrasoundenhanced delivery of targeted echogenic liposomes in a novel ex vivo mouse aorta model. J Control Release 2010, 144:288-295.

17. Michell DL, Andrews KL, Woollard KJ, Chin-Dusting JP: Imaging leukocyte adhesion to the vascular endothelium at high intraluminal pressure. J Vis Exp 2011, (54). doi:10.3791/3221.

18. Wang X, Wolf MP, Keel RB, Lehner R, Hunziker PR: Polydimethylsiloxane embedded mouse aorta ex vivo perfusion model: proof-of-concept study focusing on atherosclerosis. J Biomed Opt 2012, 17:076006.

19. AbouAlaiwi WA, Takahashi M, Mell BR, Jones TJ, Ratnam S, Kolb RJ, Nauli SM: Ciliary polycystin-2 is a mechanosensitive calcium channel involved in nitric oxide signaling cascades. Circ Res 2009, 104:860-869. 
20. Nauli SM, Kawanabe Y, Kaminski JJ, Pearce WJ, Ingber DE, Zhou J: Endothelial cilia are fluid shear sensors that regulate calcium signaling and nitric oxide production through polycystin-1. Circulation 2008, 117:1161-1171.

21. Kawanabe $Y$, Takahashi M, Jin X, Abdul-Majeed S, Nauli AM, Sari Y, Nauli SM: Cilostazol prevents endothelin-induced smooth muscle constriction and proliferation. PLoS One 2012, 7:e44476.

doi:10.1186/1480-9222-16-6

Cite this article as: Prasad et al:: Real-time vascular mechanosensation

through ex vivo artery perfusion. Biological Procedures Online 2014 16:6.

Submit your next manuscript to BioMed Central and take full advantage of:

- Convenient online submission

- Thorough peer review

- No space constraints or color figure charges

- Immediate publication on acceptance

- Inclusion in PubMed, CAS, Scopus and Google Scholar

- Research which is freely available for redistribution 